\title{
Natural Referent Vowel (NRV) framework: An emerging view of early phonetic development
}

\author{
Linda Polka, McGill University, Montreal, Canada (Linda.polka@mcgill.ca) \\ and
}

Ocke-Schwen Bohn, Arhus University, Arhus, Denmark (engosb@ hum.au.dk)

\begin{abstract}
NOTICE: this is the author's version of a work that was accepted for publication in Journal of Phonetics. Changes resulting from the publishing process, such as peer review, editing, corrections, structural formatting, and other quality control mechanisms may not be reflected in this document. Changes may have been made to this work since it was submitted for publication. A definitive version was subsequently published in Journal of Phonetics 39 (2011) 467-478, DOI: 10.1016/j.wocn.2010.08.007
\end{abstract}




\begin{abstract}
The goal of this paper is to provide an overview of an emerging new framework for understanding early phonetic development - The Natural Referent Vowel (NRV) framework. The initial support for this framework was the finding that directional asymmetries occur often in infant vowel discrimination. The asymmetries point to an underlying perceptual bias favoring vowels that fall closer to the periphery of the F1/F2 vowel space. In Polka \& Bohn (2003) we reviewed the data on asymmetries in infant vowel perception and proposed that certain vowels act as natural referent vowels and play an important role in shaping vowel perception. In this paper we review findings from studies of infant and adult vowel perception that emerged since Polka \& Bohn 2003, from other labs and from our own work, and we formally introduce the NRV framework. We outline how this framework connects with linguistic typology and other models of speech perception and discuss the challenges and promise of NRV as a conceptual tool for advancing our understanding of phonetic development.
\end{abstract}

Keywords: Vowel perception, perceptual asymmetries, Natural Referent Vowel framework, infant speech perception, phonetic development 


\section{Introduction}

In our earlier research investigating language effects on infant vowel perception we found a robust, recurring pattern (Polka \& Werker, 1994; Polka \& Bohn, 1996; 2003, Bohn \& Polka, 2001). In experiments using natural speech, we consistently observed that infant discrimination of a vowel change presented in one direction is significantly better compared to the same vowel change presented in the reverse direction. These directional asymmetries were observed when infants acquiring different languages (English and German) were tested on different minimal vowel pairs, including native and non-native contrasts. These directional asymmetries could not be explained either by considering the status of the vowel in the infant's native language (i.e. whether or not the anchor vowel is in the infant's native inventory) nor by referring to universally favored vowels (i.e. predictions based on markedness). These effects were also not consistent with an acoustic bias related to a simple increase or decrease in either F1 or F2 and could not be due to differences in amplitude, duration or pitch because these cues were not present in our stimuli. However, all of these directional asymmetries could be predicted by considering the relative position of each vowel in the vowel space defined by dimensions of vowel height (corresponding to the first formant frequency) and front/back (corresponding to the second formant frequency). Within this space, a standard metric for describing vowel acoustics and articulation, a vowel change from a more central to a more peripheral vowel is consistently easier to discriminate compared to the same change presented in the reverse direction (i.e. from a more peripheral to a more central vowel), as illustrated in Figure 1. In this figure we show vowel contrasts that have been examined in infant discrimination studies; each contrasting vowel pair is connected with an arrow indicating the direction of change that was easier to discriminate. For example, the /u/-/y/ contrast is plotted with the arrow pointing from $/ \mathrm{y} /$ to $/ \mathrm{u} /$ to indicate that the change from $/ \mathrm{y} /$ to $/ \mathrm{u} /$ was easier to discriminate than the change from $/ \mathrm{u} /$ to $/ \mathrm{y} /$. Figure 1 includes contrasts previously reviewed in Polka \& Bohn (2003) along with additional contrasts featured in subsequent research reviewed below.

Figure 1: Plot of F1/F2 frequencies for contrasts showing asymmetries in infant vowel discrimination as reviewed in Polka \& Bohn 2003 and in the present paper. Arrows point to the referent vowels for the contrast; vowel changes in this direction were easier to discriminate. Formant frequency values are approximate. ${ }^{1}$

\footnotetext{
${ }^{1}$ The * symbol on this plot represents BOTH Norwegian /i/ and Norwegian /y/ because these vowels are similar in their F1 and F2 frequencies and differ only in F3 frequency (see also footnote 8). For the sources of previously reported asymmetries, see Table 1 in Polka \& Bohn 2003. Asymmetries shown in Figure 1 and reported since Polka \& Bohn 2003 were observed in the experiments reported below in section 3.1 (for the contrasts $/ \mathrm{\Lambda} /-/ \mathrm{p} /$, /e/-/ $/$, and /e/-/e/), and in Molnar \& Polka (2004) for the /i/-/I/ contrast, see section 3.2.
} 


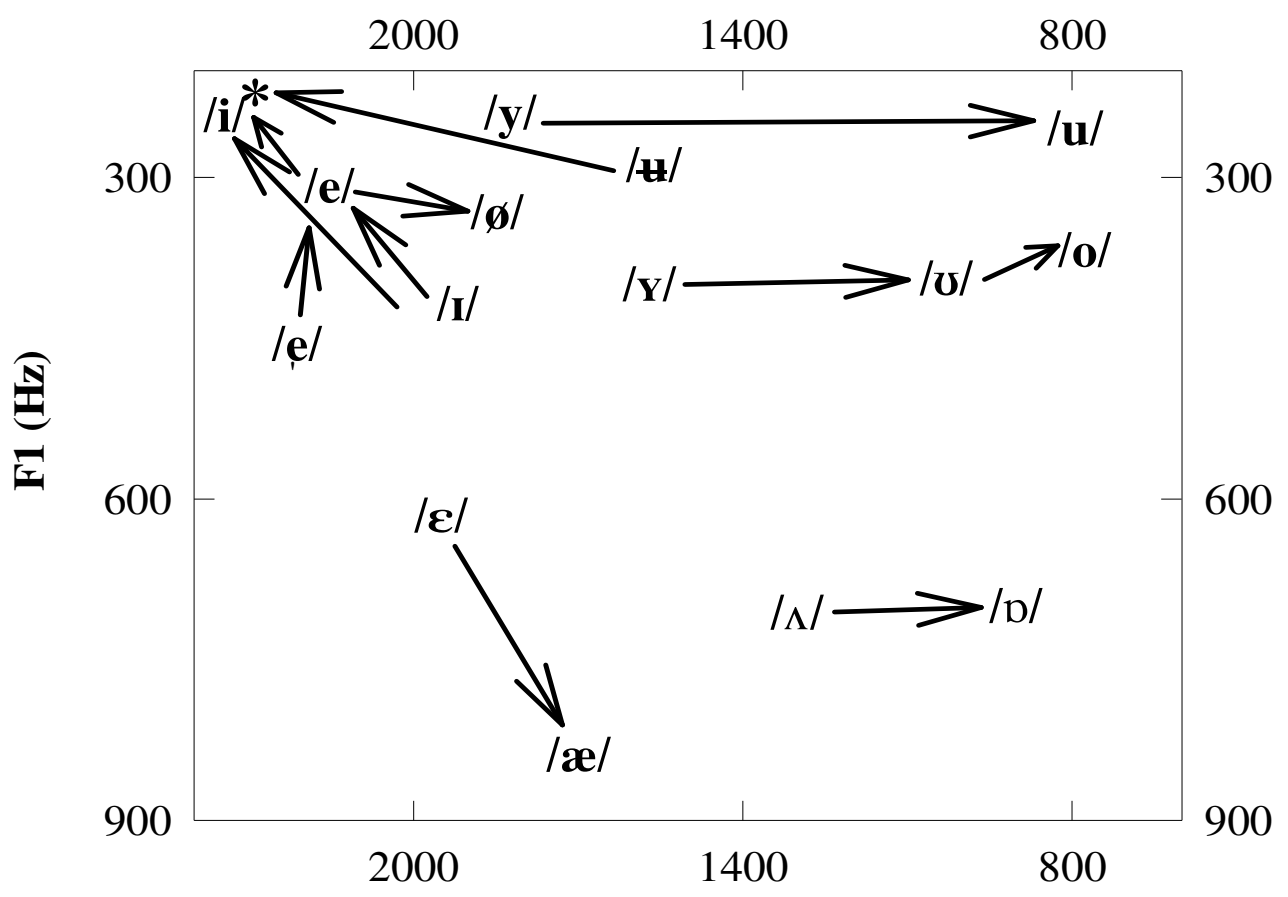

F2 (Hz)

In Polka \& Bohn (2003) we reviewed research showing directional asymmetries in vowel discrimination in infants, adults, and non-human animals (cats and birds). Asymmetries reported in a number of earlier infant studies were consistent with our hypothesis based on location within the vowel space. Overall, our survey established that asymmetries in infant vowel discrimination are robust, predictable, not dependent on specific language experience, and appear to be speciesspecific. We concluded that directional asymmetries evident in infant vowel perception point to a language-universal perceptual bias that infants bring to the task of vowel perception. These biases are either present at birth or emerge very early in development as infants begin to tune into global aspects of the speech signal.

This discovery of directional asymmetries opened up more questions and guided us to form a new approach for understanding vowel perception development, the Natural Referent Vowel (NRV) framework. The goal of this paper is to provide an update on research related to asymmetries and vowel biases conducted since Polka \& Bohn (2003) and to formally introduce NRV as a conceptual framework for understanding the development of vowel perception. Our research update includes work with adults (section 2) and with infants (section 3) and encompasses research from our labs emerging within a budding NRV framework, as well as related research in other labs. We also expand the research base with new data from several experiments with Danish adults and with Danish-learning infants. Three basic questions have guided much of the research conducted since our initial survey: 1) When and how does language experience affect asymmetries in vowel perception? 2) How well can we predict vowel asymmetries? Are they evident across areas of the vowel space that have not yet been explored? 3) What kind of processes underlie directional asymmetries? Do asymmetries reflect general (auditory) or specific (phonetic) processing biases?

In the fourth section of the paper we lay out the basic tenets of NRV and consider how this framework connects with linguistic typology and with other models of speech perception. In the 
final section we discuss the challenges and the general promise of NRV as a conceptual tool for advancing our understanding of phonetic development.

\section{Vowel perception in adults}

Adult speech perception is language-specific and well organized to optimize access to native phonemic categories. Thus, if directional asymmetries have a meaningful relationship with natural speech processing we would expect them to be affected by language experience. Accordingly, in adults we would expect asymmetries to fade or disappear for native contrasts and be maintained or enhanced for non-native contrasts. In adult speech perception language-specificity is strongest in perceptual tasks that retain some aspects of natural language processing, including high stimulus certainty and high memory load (e.g., Werker \& Logan 1985; Carney, Widin \& Viemeister, 1977). Therefore, if asymmetries are relevant in the natural speech communication contexts, then our predictions about language experience should be upheld for perceptual tasks that tap into phonetic or phonemic processing of speech and not for tasks that facilitate general auditory processing of speech.

\subsection{Danish adults' perception of English $/ \mathrm{p} /-/ \mathrm{N} /$}

A recent experiment with Danish-speaking adults provided an opportunity to evaluate our claims about language experience, and also to assess whether asymmetries emerge in other, unexplored regions of the vowel space. In this experiment, we examined L1 Danish-speaking adults' discrimination of the Southern British English (SBE) vowel contrast $/ \mathrm{p} /-/ \Lambda /$ (as in hot-hut) with a low back $(/ \mathrm{p} /)$ and a low central $(/ \Lambda /)$ vowel. Danish has no contrast in the general area of the vowel space occupied by SBE /D/-/ $/$ (Steinlen 2005, Bohn \& Bundgaard-Nielsen 2008). If directional asymmetries occur across the vowel space and are maintained in adults when the vowel contrast involved is not phonemic in the native language, then we would predict an asymmetry favoring the more peripheral vowel for this contrast in Danish adults. Specifically, discrimination of a change from the more peripheral / $\mathrm{p} /$ to more central $\Lambda /$ should be more difficult to detect than a change from $/ \Lambda /$ to $/ \mathrm{p} /$.

The stimuli for this study were selected from a database of natural CVC syllables which contain native vowels from several languages including Danish and English. For the present experiment, multiple tokens from one male native speaker of $\mathrm{SBE}$ (for $/ \mathrm{dpt} /, / \mathrm{d} \Lambda \mathrm{t} /$ ) were selected so that only dimensions relevant for vowel identity would differ across members of the vowel contrast, and that irrelevant acoustic characteristics (such as VOT, vowel duration, F0) could not be used as cues for discrimination. (Additionally, two expert listeners did not hear voice quality differences which could be used for discrimination.) Table 1 (section 3.1.2) presents the acoustic characteristics of the four tokens each for SBE /d $\Lambda \mathrm{t} /$ and /dpt/. This table shows that for the tokens for the SBE /p/$/ \Lambda /$ contrast, the only non-overlapping cue is $\mathrm{F} 2$ which clearly contrasts back $/ \mathrm{D} /$ from central $/ \Lambda /$. Figure 2 shows the position of these tokens in the F1/F2 vowel space. For ease of reference, the mean values of the extreme vowels /i/, /æ/, /a/ and /u/ are also displayed in Figure $2 .^{2}$

Fifteen Danish adults (2 male, 13 female, mean age: 26.1, SD=2.8) participated as unpaid volunteers. Selection criteria for the subjects, which were students at Arhus University, were normal hearing according to self-report, native Danish speaking parents, and minimal exposure to English (stay of less than 3 months in an English-speaking country, no English-speaking partner). We tested the adults using a go/no go discrimination task that is similar to the conditioned headturn procedure

\footnotetext{
${ }^{2}$ The mean values for these corner vowels are derived from five repetitions each of $/ \mathrm{i} / \mathrm{and} / \mathrm{u} / \mathrm{as}$ produced by the native Danish speaker (see section 3.2.1) and five repetitions each of /æ/ and /a/ by the native SBE speaker (all tokens produced in /dVt/ syllables).
} 
used in our infant experiments (see section 3.1). The listeners had to respond on each trial whether the vowel in a sequence of four / $\mathrm{dVt} /$ syllables, separated by an ISI of 2.0 seconds, had changed or not. Eighteen files were randomly presented five times: Six files were catch trials on which the vowel in the four syllables did not change (three files each with different productions of /dpt/ and of $/ \mathrm{d} \Lambda \mathrm{t} /$ ), and 12 files were change trials in which the vowel changed from $/ \Lambda /$ to $/ \mathrm{p} /(\mathrm{e} . \mathrm{g}$., $/ \mathrm{d} \Lambda \mathrm{t} / / \mathrm{d} \Lambda \mathrm{t} /$ $/ \mathrm{d} \Lambda \mathrm{t} / / \mathrm{dpt} / / / \mathrm{d} \Lambda \mathrm{t} / / \mathrm{d} \Lambda \mathrm{t} / / \mathrm{dpt} /, / \mathrm{dpt} /, / \mathrm{d} \Lambda \mathrm{t} / / \mathrm{dpt} / / \mathrm{dpt} / / \mathrm{dpt} /$ ) or from $/ \mathrm{p} / /$ to $/ \Lambda /$. All files contained four physically different /dVt/ syllables. Stimuli were presented from the hard disk of a standard PC over high-quality headphones at a self-selected comfortable listening level in a quiet environment. Subjects responded by using a mouse to click on one of two icons marked "skift" (change) and "ingen skift" (no change). Once the subjects had responded, the next trial was presented.

The adult L1 Danish listeners' responses differed significantly as a function of direction of presentation: The mean percent correct responses to a change from $/ \mathrm{p} / \mathrm{t}$ to $/ \Lambda /$ were $50.5 \% \mathrm{SD}=8.3$ ), whereas the change from $/ \mathrm{N} /$ to $/ \mathrm{p} /$ was correctly detected in $69.2 \%(\mathrm{SD}=12.1)$ of the instances $(\mathrm{t}=$ $2.54, \mathrm{df}=18, \mathrm{p}=.021$ ). The results support our prediction that asymmetries in vowel discrimination are evident in adult listeners for contrasting vowels that are not phonemic in the native language. Thus, directional asymmetries are found across the vowel space in adult perception of non-native vowel contrasts. This finding (together with the infant Experiment 1 below) shows that asymmetries present in infancy are maintained in adulthood when native language experience does not require the perceiver to interpret the vowels phonemically. ${ }^{3}$

\subsection{Adult perception of German /u/-/y/ and / $/ /-/ Y /$}

To test our hypotheses regarding language experience and examine the role of task demands we conducted a series of experiments with adults using two German vowel contrasts, /u/-/y/ and /v//Y/ (Polka, Sundara, Zhaoo, Pang, \& Ciocca, in preparation; Polka, Bohn \& Molnar 2005). For each of these contrasts we observed robust directional asymmetries in both English-learning and German-learning infants in earlier studies (Polka \& Werker, 1994; Polka \& Bohn, 1996). We used the same vowel stimuli (in / dVt/ syllables) as in these infant studies and tested adults using a go/no go task as described in 2.1. In the first experiment, we tested native monolingual English and native German-speaking adults.

The results confirmed our prediction about language experience. English adults made significantly more errors when the vowel changed from the more peripheral to the less peripheral vowel $(/ \mathrm{u} /$ to $/ \mathrm{y} /$ and $/ \mathrm{v} /$ to $/ \mathrm{Y} /)$ compared to the reverse direction. German adults tested with the same task and stimuli did not show directional asymmetries; their performance was essentially errorless in both directions, as expected for a native vowel contrast. Next we tested German and English adults on the same task and vowel contrasts using $/ \mathrm{hVp} /$ syllable productions. In this test each syllable within a 4-syllable sequence was produced by a different male talker (stimuli described in Strange, Bohn, Trent \& Nishi 2004). We obtained the same overall pattern of results as found in the single-talker condition; for both $/ \mathrm{u} /-/ \mathrm{y} /$ and $/ \mathrm{v} /-/ \mathrm{Y} /$ English adults showed asymmetries in the predicted direction and German adults' performance was near perfect and symmetrical.

Next, to explore the processes underlying these asymmetries, we tested adults in the multiple-talker condition after reducing the ISI within each syllable sequence to $500 \mathrm{~ms}$. Reducing

\footnotetext{
${ }^{3}$ Although we cannot rule out that lexical status (real word $d o t$ vs. nonword $d u t$ ) may influence the discrimination of dot-dut, we are confident that asymmetries in vowel perception are not caused by word status. First, for all vowel contrasts examined in infants and in adults, adults show the same asymmetries as prelingual infants, who do not yet have a lexicon. Second, asymmetries were found for adult listeners irrespective of whether the syllables in a contrast have word status (as the German onomatopoia /dut/ and /dyt/) or not (as the German nonwords /hup/ and /hyp/).
} 
NOTICE: this is the author's version of a work that was accepted for publication in Journal of Phonetics. A definitive version was subsequently published in Journal of Phonetics 39 (2011) 467-478, DOI: 10.1016/j.wocn.2010.08.007

the ISI has been shown to increase access to auditory memory and reduce demands on phonetic encoding (Werker \& Logan, 1985, Werker \& Tees 1984). We hypothesized that asymmetries reflect a phonetic processing bias, rather than a general auditory processing bias. Thus, we expect the asymmetries observed in English adults to be weak or absent in the reduced ISI conditions. The results confirmed this prediction; overall performance was better and neither German nor English adults showed a directional asymmetry when the ISI was reduced.

As a further step we also tested native Cantonese-speaking adults on $/ \mathrm{u} /-\mathrm{y} /$ and $/ \mathrm{v} / \mathrm{-} / \mathrm{Y} /$ contrasts using the same $/ \mathrm{hVp} /$ stimuli (multiple talkers). Cantonese is a syllable-timed language, unlike English and German which are stressed-timed languages. Moreover, in Cantonese the /u/-/y/ contrast is phonemic but $/ \mho /-/ Y /$ is not phonemic (Bauer \& Benedict 1997). If vowel perception biases are related to experience with a stress-timed language in which vowel reduction tends to be a prominent feature of the speech rhythm, then predictions regarding vowel asymmetries based on phonemic inventory alone may not be upheld across rhythmically different languages.

A standard AX task was used to test two groups of Cantonese adults on the German contrasts. One group had received training in phonetic transcription and another group had not. In the untrained group an asymmetry in the predicted direction was found for the non-native / $/ / \mathrm{V} /$ contrast but not for the native /u/-/y/ contrast. No asymmetries were found in the phonetically trained group for either contrast. These findings confirm that asymmetries are observed across subjects acquiring rhythmically different languages and are not limited to tasks involving syllable repetition. The results also show that a bias favoring peripheral vowels can be modified by training that facilitates attention to phonetic detail, consistent with our claim that asymmetries reflect a phonetic rather than a general auditory processing bias.

\subsection{Other findings on asymmetries in adult vowel perception}

Since Polka \& Bohn (2003) research from several other labs has strengthened our claim that peripheral vowels have a special status in vowel perception. This work has also provided further insights into the role of this bias in adult vowel perception.

Nishi, Strange, Akahane-Yamada, Kubi \& Trent-Brown (2008) compared the acoustic and perceptual similarity of Japanese and American English vowels. They reported that the one-mora Japanese vowels [e] and [a] were spectrally similar to $\mathrm{AE}[\mathrm{I}]$ and $[\Lambda]$. AE listeners, however, assimilated Japanese [e] and [a] not to the spectrally most similar vowels [I] and $[\Lambda]$ (which are relatively central) but to the more peripheral AE response categories [eI] and [a: o:]. Nishi et al. suggest that this result can be accounted for in terms of a perceptual bias toward more peripheral vowels, as predicted in the NRV framework. These findings suggest that default vowel perception biases observed in infancy may resurface in adults as they learn to map out a new vowel inventory in a second language.

Karypidis (2007) examined discrimination of acoustic variants surrounding each of the 10 French oral vowels by French speaking adults. For each vowel, he synthesized a prototype (P) and four "satellite" tokens (N1, N2, N3, N4) positioned at the endpoints of a cross extending 0.4 Barks from the prototype in four directions of the F1/F2 space. Discrimination of same (P/P or N1/NI) and different $(\mathrm{P} / \mathrm{N} 1, \mathrm{P} / \mathrm{N} 2$ etc) pairs was examined using an $\mathrm{AX}$ task with $500 \mathrm{~ms}$ ISI. Karypidis found that differences in the discriminability of the stimulus pairs was best accounted for in terms of the NRV framework, which predicts that discrimination of a prototype and a satellite will depend on the relative location (more peripheral vs more central) of the tokens.

\footnotetext{
${ }^{4}$ Because the distinction between [a:] and [o:] is neutralized for many AE listeners, Nishi et al. pooled responses to these two vowels as [a: 0:].
} 
Several studies conducted by Sebastián-Gallés and her colleagues add importantly to our knowledge of perceptual asymmetries in terms of both level of processing and neurophysiological correlates of perceptual biases. Sebastián-Gallés, Echeverria \& Bosch (2005) examined the ability of Catalan-Spanish bilinguals to detect mispronunciations in words containing the Catalan-only vowel / $/ \varepsilon /$, and in words containing the vowel /e/, which is shared by Spanish and Catalan. A lexical decision task revealed that bilinguals frequently did not detect mispronunciations in which $/ \varepsilon /$ replaced the correct /e/, but hardly ever made the reverse error, namely, accepting an /e/ for $/ \varepsilon /$ mispronunciation. Sebastián-Gallés et al. (2005) discuss several possible explanations for this asymmetry, ${ }^{5}$ but in the present context it is noteworthy that the direction of the asymmetry, observed at a lexical level, is as predicted by our model.

Larsson, Vera-Constán, Sebastián-Gallés \& Deco (2008) conducted a follow-up study with two experiments involving native Catalan listeners. The first of these was a replication of the lexical decision task as in Sebastián-Gallés et al. (2005). Using the same materials as in the earlier study, Larsson et al. (2008) found the same asymmetry for Catalan listeners as Sebastián-Gallés et al. (2005) had found for Catalan-Spanish bilinguals. The second experiment in the Larsson et al. (2008) study used an oddball task similar to the one we used for our adult listeners (see sections 2.1 and 2.2) to assess Catalan listeners' discrimination of the native /e/-/ع/ contrast (in /dV/ syllables). Larsson et al. used a short ISI (ca. 400ms), which favors access to auditory memory and reduces demands on phonetic encoding (Werker \& Logan 1984). They found no asymmetry for the /e/-/ع/ contrast in this task using a short ISI, consistent with our hypothesis that asymmetries reflect a phonetic processing bias, rather than a general auditory processing bias. Together, the SebastiánGallés et al. (2005) and the Larsson et al. (2008) studies confirm that perceptual asymmetries of the type described in our framework do not reflect low-level general-auditory processing, but rather higher-level processing at the linguistic phonetic and lexical levels.

A recent study by Vera-Constán \& Sebastián-Gallés (2008a) further explored this perceptual asymmetry at the lexical level, this time examining differences in reaction time and in EventRelated Potentials (ERPs) to mispronunciations of Spanish words containing substitutions of /e/ for /i/ and /i/ for /e/. Vera-Constán \& Sebastián-Gallés reported that reaction times were faster for nonwords containing the more peripheral vowel /i/ than for nonwords containing /e/, indicating that the presence of /i/ facilitated lexical decision. The ERP results were consistent with the behavioral measures in that a difference between words and nonwords appeared earlier for nonwords containing /i/ (as opposed to /e/).

Neurophysiological correlates of perceptual asymmetries were further explored by VeraConstán \& Sebastián-Gallés (2008b), who used an oddball paradigm to examine differences in mismatch negativity (MMN) between changes from /di/ syllables to /de/ syllables and changes in the reverse direction (/de/ to /di/). Consistent with their earlier results and with our model, VeraConstán \& Sebastián-Gallés found larger MMN amplitudes and a reduced latency when /di/ was the deviant stimulus interrupting a stream of /de/s than when the switch occurred from $/ \mathrm{di} /$ to $/ \mathrm{de} /$.

Overall, these recent studies confirm and extend our understanding of perceptual asymmetries in adults. The Nishi et al. (2008) study shows that the bias favoring peripheral vowels can emerge in the perceptual assimilation of nonnative vowels, and Karypidis (2007) has shown that this bias influences within-category vowel discrimination. The studies by Sebastián-Gallés and colleagues confirm that asymmetries in vowel perception are not a general-auditory phenomenon, but are due to higher level phonetic processing. In tasks involving lexical processing, these asymmetries can even be observed in native listeners. Finally, perceptual asymmetries, as predicted

\footnotetext{
${ }^{5}$ One of these is that Catalan-Spanish bilinguals are very likely to hear native Spanish-accented Catalan in which Catalan / $/ \varepsilon /$ is replaced with /e/, whereas it is less likely that /e/ is replaced with / $/ \varepsilon /$.
} 
by our model, are reflected in shorter reaction times for a more peripheral vowel in a lexical decision task, and, importantly, also in neurophysiological measures indicating a special status for peripheral vowels.

\section{Infant vowel perception}

Taken together, infant and adult findings show that directional asymmetries are shaped by language experience. Universal directional asymmetries present in infancy are retained in adult listeners for non-native contrasts but can be over-ridden for native contrasts. These findings raise the question of when language experience begins to modify these early language-general biases. Given that perception of phonetic segments begins to shift from a language-general to languagespecific form in the first year of life (Werker \& Tees 1984), we hypothesized that vowel perception biases will also become influenced by the native language within this same time frame. Accordingly, we predict that asymmetries for non-native contrasts will be maintained or enhanced across the first year of life, whereas asymmetries for native contrasts will fade or disappear.

\subsection{Experiments with Danish-learning infants}

To determine whether asymmetries are influenced by language experience across the first year of life we conducted three experiments with Danish-learning infants between 6 and 12 months of age. In these experiments we examined discrimination of two native Danish vowel contrasts and one non-native contrast. In doing so we were also able to assess whether directional asymmetries are evident in infants for two types of vowel contrasts which had not previously been examined, namely, a contrast from the low back portion of the vowel space, and a contrast which primarily differs with respect to lip rounding. All previously examined contrasts differed only with respect to the front-back and/or high-low dimension (see Figure 1).

In Experiment 1, we tested infant discrimination of the Southern British English $/ \mathrm{p} /-/ \mathrm{N} /$ contrast; this vowel pair (described above in section 2.2, table 1 and figure 2) contrasts the low back $/ \mathrm{D} /$ with a low central $/ \mathrm{N} /$ vowel. Recall that we found directional asymmetries when we tested Danish adults on this non-native contrast; as predicted discrimination of the change from more peripheral $/ \mathrm{p} /$ to more central $/ \Lambda /$ was more difficult compared to the reverse direction. We predicted that Danish infants, like Danish adults, will maintain a directional asymmetry across the first year of life because their language does not make use of this contrast to form words.

In Experiment 2, we examined the discrimination of the contrast between the second and the third highest front unrounded Danish vowels, which are represented in Danish orthography as <e> and $\langle\mathfrak{x}\rangle$. The IPA representation of these vowels would be /e/ with a diacritic indicating raising, and /e/ with a diacritic indicating lowering. For reasons of convenience and legibility, these vowels will be represented here as /e/ and / $/$ /, but it should be borne in mind that what is labeled here as Danish / $\varepsilon /$ is much closer to Cardinal Vowel 2 than to Cardinal Vowel 3. We predicted that young infants will show a directional asymmetry favoring the more peripheral vowel for this contrast; specifically, discrimination of a change from /e/ to / $/ \varepsilon /$ should be more difficult to detect than a change from $/ \varepsilon /$ to $/ \mathrm{e} /$. However, since this is a native contrast, experience with this contrast in the ambient language may attenuate the asymmetry as infants approach their first birthday.

In Experiment 3 we examined the Danish /e/-/ø/ contrast between two front and high vowels which differ primarily in that /e/ is unrounded whereas / $\varnothing /$ is rounded. In addition, the vowels differ in that /e/ is slightly more front compared to the more central / $\varnothing /$. For this contrast we again predict an asymmetry favoring the more peripheral vowel /e/; specifically discrimination of a change from /e/ to / $/$ / should be more difficult to detect than a change from $/ \varnothing /$ to /e/. As for the native /e/-/ع/ contrast, the Danish infants' experience with /e/-/ø/ may attenuate the asymmetry in the first year. 


\subsubsection{General Methods for Experiments 1-3 with Danish-learning infants}

Stimuli: The stimuli for our three experiments were selected from the same database mentioned in section 2.2. For Experiment 1 we used the same syllables as in the adult experiment described in section 2.2. Applying the same criteria used to choose the English /dpt/ and $/ \mathrm{d} \Lambda \mathrm{t} /$ tokens, we selected multiple tokens from one male native Danish speaker (for /det, døt, det/) and one male native speaker of SBE (for /dpt/, /d $\Lambda \mathrm{t}$ ). Table 1 presents the acoustic characteristics of the Danish $/ \mathrm{dVt} /$ syllables used in the experiments. For the Danish /det/-/det/ tokens the only reliable cue is F1 signalling the difference between the higher, more peripheral /e/ and the somewhat lower and less peripheral /ع/. The tokens for the Danish /det/-/døt/ contrast differ both with respect to F2 and F3, with /e/ being more peripheral in the F1/F2 space than / $\varnothing /$. Figure 2 shows the position of the Danish vowel tokens used in Experiment 2 and 3 in the F1/F2 vowel space.

Table 1: Acoustic characteristics of the stimuli used in experiments with Danish -speaking and Danish-learning infants. Experiments 1-3. V dur: vowel duration, VOT $=$ voice onset time, F0 = fundamental frequency, F1, F2, F3 = frequencies of the first three formants. Measurements of F0 and F-F3 were made near the vowel midpoint.

\begin{tabular}{|l|l|l|l|l|l|l|}
\hline Token & V dur & VOT & F0 & F1 & F2 & F3 \\
\hline d $\Lambda t-1$ & 151 & 10 & 125 & 705 & 1433 & 2319 \\
\hline d $\Lambda \mathrm{t}-2$ & 149 & 11 & 111 & 651 & 1460 & 2380 \\
\hline d $\Lambda \mathrm{t}-3$ & 173 & 11 & 125 & 654 & 1490 & 2278 \\
\hline $\mathrm{d} \Lambda \mathrm{t}-4$ & 135 & 11 & 142 & 679 & 1434 & 2726 \\
\hline dpt-1 & 152 & 11 & 100 & 660 & 876 & 2461 \\
\hline dpt-2 & 172 & 12 & 125 & 662 & 866 & 2482 \\
\hline dpt-3 & 189 & 9 & 111 & 620 & 960 & 2604 \\
\hline dpt-4 & 172 & 12 & 125 & 668 & 889 & 2380 \\
\hline det-1 & 98 & 29 & 111 & 349 & 1901 & 2588 \\
\hline det-2 & 104 & 25 & 125 & 315 & 1856 & 2632 \\
\hline det-3 & 99 & 28 & 111 & 349 & 1879 & 2509 \\
\hline det-1 & 106 & 20 & 111 & 405 & 1823 & 2498 \\
\hline det-2 & 117 & 20 & 125 & 416 & 1879 & 2610 \\
\hline det-3 & 100 & 22 & 111 & 416 & 1811 & 2542 \\
\hline døt-1 & 100 & 23 & 125 & 360 & 1597 & 2239 \\
\hline døt-2 & 101 & 27 & 125 & 349 & 1620 & 2261 \\
\hline døt-3 & 81 & 34 & 125 & 338 & 1642 & 2351 \\
\hline
\end{tabular}


Figure 2: F1/F2 plot of the vowels used in Experiments 1-4 (see text). The corner vowels /i, u, æ, a/ are represented by the means of five tokens each.

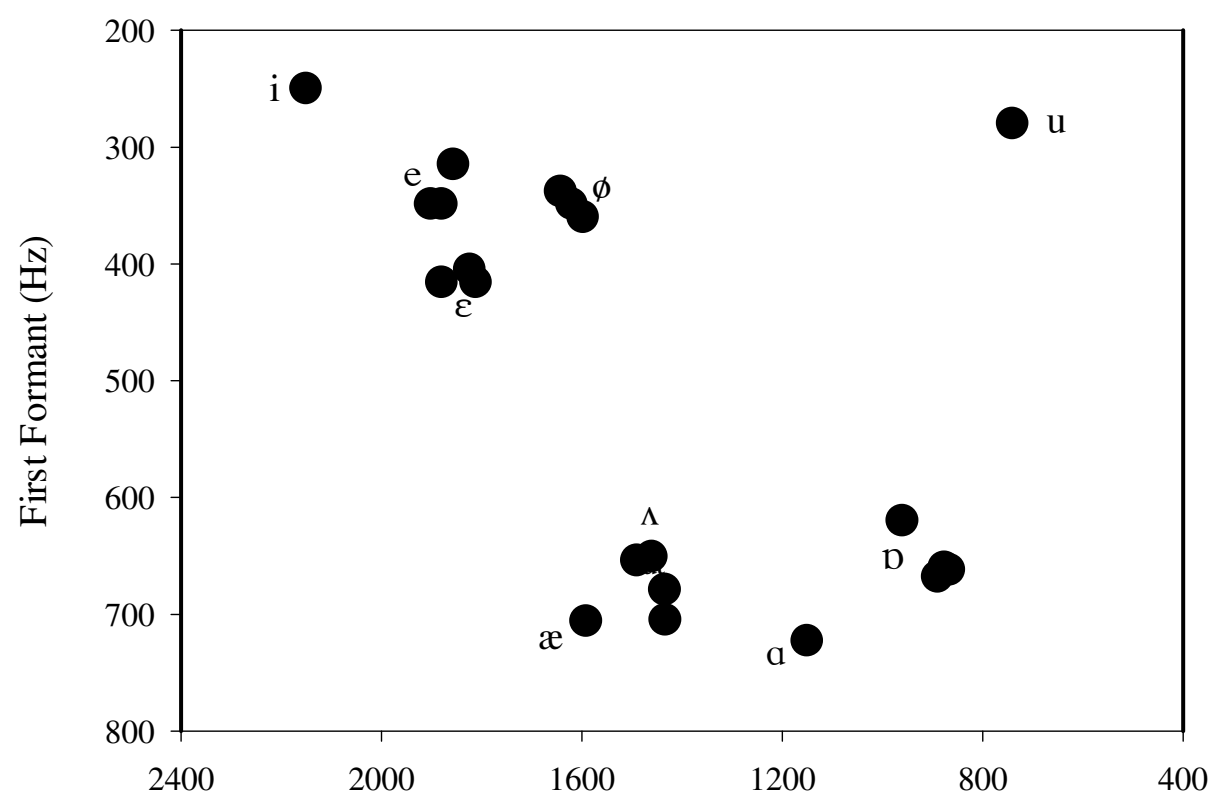

Second Formant $(\mathrm{Hz})$

Subjects: One hundred and ninety-nine infants (101 males, 98 females) between the ages of 6 to 12 months served as subjects. All were healthy, full-term infants with no history of ear infection (by parental report). All infants were being raised in monolingual Danish-speaking families in the Arhus region of Denmark. The infants were randomly assigned to the three experiments, and within experiments, infants were randomly assigned to directions of presentations (peripheral vowel to central vowel, or central to peripheral).

Procedure: Infants were tested in the conditioned headturn procedure. We used the same experimental equipment and setup and largely the same procedures as in Polka \& Bohn (1996) and Bohn \& Polka (2001), to which we refer for details. Briefly, a stream of syllables (1500 ms ISI) was played from a loudspeaker. At random intervals the background syllables were changed to foreground syllables for a 4.8 second interval during which three tokens were presented which were drawn from the same category as the background syllables (control trial) or from a contrasting category (change trial). The presentation of members of a contrast as background or as foreground is counterbalanced in the headturn procedure, which provides an opportunity to examine perceptual asymmetries. Background and target consisted of multiple tokens of each vowel category, played in random order.

Each infant completed either one or two test sessions conducted on different days. Each infant test session consisted of two conditioning stages and a testing stage. In the first conditioning stage, the infant was trained to respond to a change from either $/ \mathrm{dit} /$ (for the SBE $/ \mathrm{p} /-/ \mathrm{N} /$ contrast) or /dat/ (for the Danish /e/-/ø/ and /e/-/ع/ contrasts) to the syllable that will be presented as foreground during the testing stage. Each test stage included 25 trials with ca. 15 change and ca. 10 control (nochange) trials. As in our previous studies, we used as conjunct criteria for successful discrimination; infants had to achieve a per cent correct score of $>60 \%$ (hits for change trial plus correct rejections 
for control trials) and at least 7/8 consecutive correct responses. We only used data from infants who either reached criterion in the first test session (testing discrimination of either $/ \mathrm{p} /-/ \mathrm{N} /$, /e/-/ø/, or $/ \mathrm{e} /-/ \varepsilon /$ ) or, if they failed to do so, reached criterion in a second session (testing discrimination of the extreme contrast /i/-/a/). This second session helped us determine whether the contrast (and direction of presentation) in the first session was too difficult (for those infants who did not reach criterion in the first session, but discriminated /i/-/a/ in he second session), or if some other undetermined cause was responsible for the infant not reaching criterion (if the infant did not discriminate the extreme contrast /i/-/a/.

\subsubsection{Experiment 1: Danish infants on non-native (SBE) hot-hut}

Seventy Danish-learning infants $(32 \mathrm{~m}, 38 \mathrm{f})$ between the ages of $6: 15$ and 11:20 months were tested on Southern British English contrast $/ \mathrm{D} /-/ \Lambda /{ }^{6}$ For the 35 infants who heard the background vowel $/ \mathrm{p} /$ changing to the foreground $/ \Lambda /$, the mean correct discrimination score was $56.2 \%(\mathrm{SD}=10.5)$. For the 35 infants who heard a change from the background vowel $/ \Lambda /$ to the foreground $/ \mathrm{p} /$, mean correct discrimination was $64.3 \%(\mathrm{SD}=9.6)$. The significant difference in per cent correct discrimination between the directions of presentations $(\mathrm{t}=2.811$, df $=68, \mathrm{p}=.001$ ) supports the prediction that a change from a more peripheral vowel to a less peripheral vowel is more difficult to discriminate than a change in the opposite direction. The result also shows that a perceptual bias favoring relatively peripheral vowels extends to the low back portion of the vowel space.

To examine whether the perceptual asymmetry was affected by the age of the infants, we separately analyzed the results for the 34 younger infants (aged between 6:15 and 8:21 months) and the 36 older infants (aged between 8:25 and 11:20 months). The percent correct scores for the younger infants were, for the $/ \mathrm{p} /->/ \Lambda /$ direction of presentation, $55.7 \%(\mathrm{SD}=8.7)$, and for the $/ \Lambda /->$ $/ \mathrm{p} /$ direction, $63.4 \%(\mathrm{SD}=9.7), \mathrm{t}=2.441(\mathrm{df}=32), \mathrm{p}=.02$. The percent correct scores for the older infants were, for the $/ \mathrm{p} / \rightarrow / \Lambda /$ direction of presentation, $56.7 \%(\mathrm{SD}=12.2)$, and for the $/ \Lambda /->/ \mathrm{p} /$ direction, 65.1\% ( $\mathrm{SD}=9.8), \mathrm{t}=2.263(\mathrm{df}=34), \mathrm{p}=.03$. These results are consistent with the NRV prediction that the perceptual asymmetry will be maintained for non-native vowel contrasts.

\subsubsection{Experiment 2: Danish infants on native $/ \mathrm{e} /-/ \varepsilon /$}

Seventy-three Danish-learning infants $(37 \mathrm{~m}, 36 \mathrm{f})$ between the ages of 6:10 and 11:28

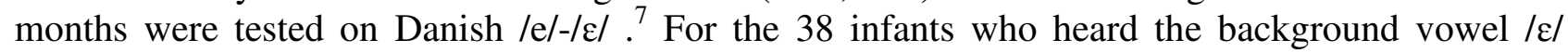
changing to the foreground /e/, the mean correct discrimination score was $59.7 \%$ (SD $=11.5$ ). For the 35 infants who heard a change from the background vowel /e/ to the foreground / $/$, mean correct discrimination was $53.9(\mathrm{SD}=10.4)$. The significant difference in per cent correct discrimination between the directions of presentations $(\mathrm{t}=2.264, \mathrm{df}=71, \mathrm{p}=.027)$ supports the NRV prediction that a change from a more peripheral vowel to a less peripheral vowel is more difficult to discriminate than a change in the opposite direction.

To examine whether the perceptual asymmetry was affected by the age of the infants, we separately analyzed the results for the 30 youngest infants (aged between 6:10 and 9:02 months) and the 30 oldest infants (aged between 10.14 and 11:28 months). The percent correct scores for the

\footnotetext{
${ }^{6} 69$ additional infants were tested but failed to provide complete data for the following reasons: failure to condition in the first session (13), failure to reach criterion in the first session and in a second session testing discrimination of /i/-/a/ (44), scheduling problems (10), and experimenter error/equipment failure (2).

730 additional infants were tested but failed to provide complete data for the following reasons: failure to condition in the first session (3), failure to reach criterion in the first test session and in a second session testing discrimination of /i//a/ (19), and scheduling problems (8).
} 
30 youngest infants were, for the $/ \varepsilon /->/ \mathrm{e} /$ direction of presentation, $60.1 \%$ (SD=8.6), and for the /e/ $\rightarrow / \varepsilon /$ direction, $51.1 \%(\mathrm{SD}=11.8), \mathrm{t}=2.39(\mathrm{df}=28), \mathrm{p}=.024$. The percent correct scores for the 30 oldest infants were, for the $/ \varepsilon /->/ e /$ direction of presentation, $60.9 \%(\mathrm{SD}=12.1)$, and for the /e/ $->$ $/ \varepsilon /$ direction, 57.9\% $(\mathrm{SD}=7.8), \mathrm{t}=.825(\mathrm{df}=28), \mathrm{p}=.416$. These results are consistent with the NRV prediction that experience with a vowel contrast will override the initial default bias favoring peripheral vowels.

\subsubsection{Experiment 3: Danish infants on native /e/-/ø/}

Fifty-six Danish-learning infants $(32 \mathrm{~m}, 24 \mathrm{f})$ between the ages of 6:0 and 11:9 months were tested on Danish /e/-/ø/. ${ }^{8}$ The difference in per cent correct discrimination between the 30 infants who heard the background vowel / $\varnothing /$ changing to the foreground /e/ $(58.5 \%, \mathrm{SD}=11.2)$ and the 26 infants who heard a change from the background vowel /e/ to the foreground $/ \varnothing /(58.0 \%, \mathrm{SD}=11.1)$ was nonsignificant $(\mathrm{t}=.183, \mathrm{df}=54, \mathrm{p}=.856)$. However, this result masks differences between the younger and the older infants in this experiment. For the 24 youngest children (age range: 6:0-7:16), the direction of presentation significantly affected the discriminability of the /e/-/ø/ contrast, albeit in a direction opposite from our prediction. For these infants, a change from the less peripheral $/ \varnothing /$ to the more peripheral /e/ $(54.4 \%$ correct, $\mathrm{SD}=6.9)$ was significantly less discriminable than a change from $/ \mathrm{e} /$ to $/ \varnothing /(61.8 \%$ correct, $\mathrm{SD}=9.8), \mathrm{t}=2.126(\mathrm{df}=22), \mathrm{p}=.045$. For the 24 oldest infants (age range: 7:22-11:9), however, the direction of presentation did not significantly affect discriminability from $/ \varnothing /$ to $/ \mathrm{e} /$, even though the discrimination scores suggest a reversal of the asymmetry. The scores for these children were, for the $/ \varnothing /->/ \mathrm{e} /$ direction of presentation, $63.3 \%$ correct $(\mathrm{SD}=13.2)$, and for the $/ \mathrm{e} /-/ \varnothing /$ direction, $55.8 \%$ correct $(\mathrm{SD}=12.1), \mathrm{t}=1.438$, $\mathrm{df}=22, \mathrm{p}$ $=.164$.

Experiment 3 provided the first counterevidence to our prediction that, within the F1/F2 vowel space, perception will be biased to favor the relatively more peripheral vowel. However, the results from this experiment are consistent with our prediction that experience with a vowel contrast will attenuate any perceptual asymmetry.

\subsubsection{Discussion}

With one exception, the results of Experiment 1-3 were consistent with the our predictions: The asymmetry for the non-native $/ \mathrm{p} /-/ \Lambda /$ was maintained throughout the second half of the first year of life, whereas asymmetries for native contrasts $(/ \mathrm{e} /-/ \varepsilon /$ and $/ \mathrm{e} /-/ \varnothing /)$ were only evident in younger infants. As predicted, older infants did not show a perceptual bias for the relatively more peripheral vowel in the native contrasts, suggesting that attunement to the L1 led to an attenuation of the default bias favoring peripheral vowels. These findings are consistent with our prediction that specific vowel experience will override the perceptual default bias favoring relatively peripheral vowels, and that asymmetries will be maintained only of listeners lack experience with specific vowels.

Our predictions were not met with respect to discrimination of the native /e/-/ø/ contrast in the younger infant group. Contrary to previous findings, the directional asymmetry for this Danish vowel contrast did not favor /e/ which is more peripheral than / $\varnothing /$ in the F1/F2 acoustic space. This outcome shows that we cannot predict all asymmetries based on the location of vowels within in a simple F1/F2 vowel space. We clearly need to re-consider why these effects occur and what parameter underlies directional asymmetries.

\footnotetext{
${ }^{8} 20$ additional infants were tested but failed to provide complete data for the following reasons: failure to condition in the first session (4), failure to reach criterion in the first test session and in a second session testing discrimination of /i//a/ (8), scheduling problems (6), and experimenter error/equipment failure (2).
} 
This unexpected asymmetry for Danish /e/-/ø/ involves a vowel contrast that differs in liprounding, whereas most of the contrasts showing a directional asymmetry differ in vowel height and/or front/back dimensions. To date, one other study has shown an asymmetry that cannot be predicted based on location within the F1/F2 vowel space. It is noteworthy that this contrast also involves a difference in lip-rounding. Best \& Faber (2000) examined the discrimination of two Norwegian vowels, traditionally transcribed as /i/ and /y/; these vowels are high and front, but their articulatory descriptions differ in that $/ \mathrm{i} /$ is unrounded and $/ \mathrm{y} /$ is outrounded. ${ }^{9}$ Best \& Faber tested discrimination of this vowel pair by English-learning infants at several ages. Infants in the youngest age group (3-5 months of age) discriminated the contrast in the /i/ to /y/ direction, but not in the /y/ to /i/ direction. ${ }^{10}$ Similar to Danish /e/-/ $\varnothing /$, the asymmetry observed for Norwegian /i/ to /y/ cannot be predicted on the basis of F1 and F2 alone (neither formant differs across these two vowels) and likely involves information present in F3 or higher formants. For these Norwegian vowels it is the rounded vowel that acts as a referent, again similar to the asymmetry observed for Danish /e/-/ø/.

These unexpected findings suggest that understanding asymmetries for vowels that contrast in lip-rounding will lead us a more principled account for directional asymmetries. Lip rounding lowers all formant frequencies with strong effects on F3 and higher formants. Assuming that perceptual asymmetries have a common basis, we need to consider additional formants (F3 and possibly F4) not just the location of F1 and F2 in a simple two-dimensional space. We propose that multiple formants (minimally F1, F2, and F3) and their spectral proximity (which also effects formant amplitude or focalization) must be considered to account for asymmetries in a comprehensive way. The acoustic patterns that underlie directional asymmetries are discussed further in section 4 below.

\subsection{Directional asymmetries and infant perceptual biases}

Directional asymmetries appear to be a robust and predictable feature of infant vowel perception. One interpretation of these effects is that peripheral vowels provide a stable, default perceptual frame for the young perceiver. The peripheral vowels $/ \mathrm{i} /, \mathrm{a} / \mathrm{and} / \mathrm{u} /$ are universal, found in all languages of the world. Therefore an initial bias favoring vowels falling close to the corners of the vowel space is clearly advantageous no matter what language is to be acquired. This bias will direct the infant to some of the vowels that will form their native inventory and can also provide initial reference points to organize vowel perception. While this explanation is compelling, we need more direct evidence that infants selectively attend or process more peripheral vowels to support this interpretation.

To test our claim that peripheral vowels are prioritized in infant speech processing we tested infant listening preferences for two vowel contrasts (Molnar \& Polka, 2004; Polka \& Molnar (in preparation). One contrast, German $/ \mathrm{u} / \mathrm{vs} / \mathrm{y} /$, had shown a robust directional asymmetry in discrimination for both native (German) and non-native (English) babies in our earlier research (Polka \& Werker, 1994; Polka \& Bohn, 1996). The other contrast was English /i/-/I/. Directional asymmetries were reported for this contrast in infant research using synthetic speech that varied in formant frequencies but not duration (Swoboda, Morse \& Leavitt 1976; Swoboda, Kass, Morse \& Leavitt 1978). For this contrast we used several /hid/ and /hId/ syllables produced by two female talkers from a corpus of English vowels developed by Hillenbrand, Getty, Clark, \& Wheeler (1995).

\footnotetext{
${ }^{9}$ It is important to note that the Norwegian /y/ is quite different from the /y/ vowels of Danish or German. Both the acoustical measurements for Norwegian /y/ in the Best and Faber (2000) study (kindly provided by C. Best, p.c.), and values reported by Kristoffersen (2000) suggest that Norwegian /i/ and /y/ do not differ with respect to the front-back dimension (with F2 values for these vowels differing by .35\% according to Kristoffersen (2000), and by $2 \%$ for the Best $\&$ Faber study). Both studies report slightly lower F1 values for $/ \mathrm{y} /$ than for $/ \mathrm{i} /$.

${ }^{10}$ The 6-8 months olds in the Best \& Faber (2000) study failed to discriminate in both directions, and the 10-12 months old discriminated in both directions.
} 
NOTICE: this is the author's version of a work that was accepted for publication in Journal of Phonetics. A definitive version was subsequently published in Journal of Phonetics 39 (2011) 467-478, DOI: 10.1016/j.wocn.2010.08.007

These natural $/ \mathrm{hVd} /$ syllables were resynthesized using sinusoidal synthesis, an excellent technique for preserving the spectral properties of vowels; the re-synthesized tokens that were essentially indistinguishable from natural productions (see Hillenbrand, Clark \& Houde 2000 - experiment 2). Duration differences between /hid/ and /hId/ were neutralized in these stimuli.

We presented these two contrasts to infants in a sequential preferential listening task implemented using the headturn preference paradigm (Jusczyk \& Aslin 1995). We tested infants from monolingual families who were acquiring either English or French, and also infants from bilingual families who were acquiring French plus another language or English plus another language. The /u/- /y/ contrast is phonemic in French but not in English; the reverse applies to /i/-/I/ contrast, i.e. it is phonemic in English but not in French. For each language group infants were tested in 3 age groups: 3-5 months, 6-8 months, and 10-12 months. Separate preference tests were conducted for each contrast.

We predicted that infants would prefer /dut/ when tested with /dut/ and /dyt/ syllables and would prefer /hid/ when tested with /hid/ and /hid/ syllables. If directional asymmetries reflect an underlying universal bias favoring peripheral vowels we expected to find the predicted preferences across the 3 language groups, at least in the younger infants. If language experience begins to shape this default bias during infancy, then an interaction between listening preference and language group may emerge with age as the size of the preference diminishes in the native group but not in the non-native group. This is what we found for monolingual groups. For both monolingual English and monolingual French infants at all 3 ages, the predicted preferences for /dut/ and for /hid/ were observed. These preferences were robust and present in almost every baby tested. An interaction between preference and language also emerged with age for /dut/-/dyt/; the magnitude of the /dut/ preference was smaller in the French infants compared to the English infants among the 10-12 months olds. No language interaction was evident for /hid/-/hId/.

We included bilingual infants to extend the interpretation of our finding. If directional asymmetries reflect a universal, language-independent bias then both monolingual and bilingual infants should show asymmetries early in development. Moreover, if language experience affects these biases in the first year of life, we could expect bilingual and monolingual infants to show a different pattern of change with increasing age, given the differences in their language experience vis-à-vis the two vowel contrasts. Surprisingly, for both contrasts we found robust directional asymmetries in the bilingual 6- to 8-month-olds and 10-12-month-olds, but not in 3- to 5-montholds. Thus experience with two languages clearly affected the early appearance of these vowel biases. The peripheral bias may emerge in the first few months of life in monolingually-exposed infants and appear a bit later in bilingually-exposed babies. Alternatively, the bias may be present at birth but then fade and re-emerge in bilingual infants; the later pattern has been observed in bilingual infant vowel discrimination (Bosch \& Sebastián-Gallés 2003). Further research with younger infants will clarify which interpretation is correct. In either case, our bilingual findings confirm that infant vowel preferences are affected by exposure to a mixed language input and hence reflect a speech processing bias, not a simple acoustic processing bias. The preference findings also confirm that directional asymmetries indeed reflect any underlying perceptual bias; peripheral vowels are favored in infant perception. This is evident in individual infants in a direct test of perceptual bias.

\subsection{Asymmetries in the development of infant perception of vowel duration.}

A recent study by Mugitani et al. (2007) also shows asymmetries in vowel perception that change with language experience. In this study vowel duration alone was the critical cue whereas all previous studies showing asymmetries involve vowel quality contrasts. Mugitani and colleagues 
found that 18 month-olds acquiring English, in which vowel duration is not phonemic, show a symmetric pattern of vowel discrimination; they discriminated a vowel change in either direction (short to long or long to short). However, 18-month-olds acquiring Japanese, in which vowel duration is phonemic, show an asymmetric pattern; they discriminated a long to short vowel change but not a short to long vowel change. Younger Japanese infants (10-month-olds) show a symmetric pattern, similar to English 18-month-olds. Japanese adults show a symmetric pattern for crosscategory vowel duration differences (that were tested with infants) but they show asymmetries when discriminating smaller within-category duration differences. Japanese infants thus appear to move through a stage in which they treat the short vowel, which is more frequent and less variable compared to the long vowel in their input, as a reference or anchor. Using this reference point they organize their perception to interpret vowel duration phonemically. When the native phonology is consolidated (in Japanese adults) vowel duration discrimination becomes symmetrical for large duration differences (that distinguish long/short vowel categories) but asymmetric for smaller duration differences (that are within a vowel category). It is not clear why these findings do not line up more precisely with the age and language effects found for asymmetries in spectral vowel contrasts. There may be fundamental differences in the perception of vowel duration and quality given that vowel quality is phonemic in all languages but vowel duration is not phonemic in most languages. Nevertheless, consistent with the NRV framework, these findings show that perceptual biases change with language experience; they emerge and recede during the acquisition of a native phonemic contrast and appear to support phonological development.

\subsection{The Natural Referent Vowel Framework}

Previous findings reviewed in Polka \& Bohn (2003) and subsequent research summarized above and in Figure 3 provided the impetus for a new approach for understanding the development of vowel perception - the Natural Referent Vowel (NRV) framework. Presently, this conceptual framework is focused on understanding the development of vowel quality perception.

Within the NRV framework we propose that vowels with extreme articulatory-acoustic properties (peripheral in the vowel space) act as natural referent vowels. Natural referent vowels support and guide the development of vowel perception by attracting infant attention and providing stable perceptual forms for the language learner. This is an adaptive default bias because the peripheral vowels within the F1/F2 vowel space - /i/, /a/, /u/ - are a universal feature of the vowel inventory of all human languages. These early vowel perception biases reveal the perceptual proclivities of the human auditory system at birth, and/or perceptual learning occurring in the first few weeks or months of life. We speculate that these early vowel perception biases point to a global structure for recognizing acoustic patterns as human speech signals, similar to their early acquisition of a global schema for recognizing certain visual configurations as a face (e.g., Gliga, Elsabbagh, Andravizou \& Johnson 2009).

Language experience builds on the default pattern established by the initial vowel perception biases. Through experience with a specific language, learners access other vowel categories and organize their vowel perception to optimize native language perception. As the child gains experience with its native language, the early vowel biases favoring peripheral vowels are maintained, enhanced or over-ridden as needed to optimize access to language-specific vowel categories and contrasts. The bias favoring peripheral vowels affords access to a minimal vowel inventory given that $/ \mathrm{i} / \mathrm{/} / \mathrm{a} / \mathrm{and} / \mathrm{u} /$ are universal. This minimal set also provides important reference points that are used to discover and extract additional vowel categories. The bias favoring referent vowels will support perception of several native vowel categories but this bias alone is inadequate when a spectrally similar vowel is also functionally important in the native language. In this case, 
the perceiver can learn to go beyond the bias and access vowels that are similar to, but distinct from, a referent vowel. As a result, in mature perceivers vowel perception biases (and their related directional asymmetries) will be maintained or enhanced for non-native vowel contrasts but will be reduced or absent for native vowel contrasts in many phonetic processing tasks. With development, the learner will shift from a default bias to a perceptual system that is elaborated to accommodate the native vowel system. This shift will begin in infancy and will continue until the native phonology is consolidated.

Mature perceivers can override the initial biases as needed to access functional native vowel categories. However, the perceptual bias favoring natural referent vowels is grounded in acoustic patterns that have an easy, privileged fit with human auditory/articulatory abilities. For this reason, this bias will re-emerge in some processing conditions even for native contrasts. The Natural Referent Vowel bias may re-emerge in measures of processing speed, in the neural processing of vowels, in degraded listening conditions (e.g., noise), or when native language processing does not require a complete over-ride to support language comprehension. This bias may also re-appear when perceivers are mapping out a new vowel system to learn a second language, possibly when new L2 vowel categories fall in regions of the vowel space that are not firmly committed to the L1.

The salience and stability of natural referent vowels is due to formant frequency convergence or focalization (Schwartz, Boe, Vallée \& Abry 1997; Schwartz, Abry, Boe, Ménard $\&$ Valée 2005). As formants move close together in frequency there is a mutual reinforcement of acoustic energy that raises the amplitude of each formant. As a result, formant convergence effectively focuses energy into a narrower spectral region. Thus, the focalization resulting from formant convergence produces salient prominences in the vowel spectra. As shown by Chistovich and colleagues (Chistovich \& Lublinskaya 1979; Chistovich 1985), the human ear, which perceptually integrates formants that are spectrally close, is clearly sensitive to the spectral prominences created by formant convergence. When there is a perceptual integration of adjacent formants an effective formant is created with a center of gravity (re amplitude and spectrum) that is a weighted average of the individual formants (see also Strange, 1999).

Focalization is graded and gives rise to salience differentials across the vowel space. Focalization reaches maxima for a subset of vowels - /i/, /a/, /u/, and /y/ - that approach formant convergence limits; these are referred to as focal vowels. Three focal vowels, /i/, /a/ and /u/, fall at the corners of the traditional vowel space (defined by vowel height and front/back dimensions). ${ }^{11}$ However, the formant convergences that underlie and define /i/ / $/$ / and /a/ as focal vowels require minimally a 3-dimensional vowel space (F1-F2, F2-F3, F3-F4). As well, the status of /y/ as a focal vowel (due to convergence of F2 and F3) is not depicted in the traditional F1/F2 vowel space. For a detailed explanation of focal vowels and their description within a 3-dimension vowel space see Schwartz et al (2005).

Concurring with Schwartz et al al (2005) we propose that the perceptual prominence of natural referent vowels is a result of increased focalization. For unrounded vowels, focalization and location in the F1/F2 vowel space predict the same directional asymmetries. For vowel contrasts that involve lip-rounding, the more focal vowel within a pair may not necessary fall closer to the edge or corner of the F1/F2 vowel space. Thus, with focalization providing the underlying support for vowel perception biases the F1/F2 vowel space will not account for all vowel perception asymmetries. An expanded vowel space that incorporates three dimensions and minimally includes F3 is required. Focalization as a basis for NRV is discussed further in section 4.1.

\footnotetext{
${ }^{11}$ Vowels in which two formants converge maximally are also called quantal vowels within the Quantal Theory of speech developed by Stevens (1989).
} 
We propose that Natural Referent Vowels play an important role in early L1 acquisition. Vowel perception biases result in a rudimentary schema for interpreting vowels as a human speech signal, and establish a basic scaffold for interpreting vocalic segments, which are a prominent portion of the human speech signal. With this default perceptual framework in place the infant has the foundation for processing vowels in any language. The salience and stability of referent vowels make them easy to encode and hold in memory; this allows them to serve as targets for contrasting with less-focal vowels. These properties also allow Natural Referent vowels to function as production targets that infants can aim for to expand and organize their emerging vowel production skills (Buhr 1980; Lieberman 1980; Kent \& Murray 1982; Ishizuka, Mugitani, Kato \& Amano 2007)

The precise role of Natural Referent Vowels has not been established empirically. We speculate that NRVs may facilitate the recognition of equivalence across vowels produced by different talkers. We also speculate that NRVs guide and support the development of vowel production during infancy. Infants favor front, central vowels in the initial stages of vowel production. However, within a short period their vowel productions expand to encompass a range of vowel qualities that span both dimensions of the F1/F2 space (Buhr 1980; Lieberman 1980; Kent \& Murray 1982, Ishizuka et al. 2007). The default perceptual frame provided by the NR vowels may provide stable targets to guide the infant's acquisition of a productive vowel space and may be essential for establishing an initial perceptual/motor coupling that is critical for further development.

\subsection{Relationship of the Natural Referent Vowel framework to phonetic universals and typology}

Phonetic universals have played a prominent role in theories seeking to explain vowel system typologies that characterize human languages. As outlined in Lindblom's dispersion theory (e.g., Lindblom \& Engstrand 1989), the universality of the focal or quantal vowels, / $/ /, / a / / u /$, is important in establishing global processing constraints within a language, i.e. these vowels establish a minimal system organized so that elements are sufficiently distinct or dispersed from one another. According to Schwartz and colleagues (Schwartz et al. 1997; Schwartz et al. 2005), focal vowels provide these global constraints but are also universally favored across vowel inventories because of their intrinsic properties. They propose that focal vowels are selected because they have perceptual attributes that make them worthy phonetic elements on their own, independent of their relationship with or configuration relative to other vowels in a linguistic system. Based on their vowel perception experiments with adults, Schwartz \& Escudier (1989) propose that focal vowels are "good" auditory patterns and are more stable in short-term memory when compared to non-focal vowels. This view was elaborated in a later paper outlining their dispersion-focalisation theory of vowel systems in which they concluded that

... formant convergence could result in increased "perceptual value" for a given spectral configuration because of acoustic "salience". This "focal" quality can be related to "focal colors" (Brown \& Lenneberg, 1954) for which there seems to be general (cross-linguistic) perceptual agreement (Rosch-Heider, 1972). Hence, Lieberman (1971, pp 57-58) notices that "quantal sounds provide acoustic salience; that is, their formant frequency patterns yield prominent spectral peaks ... that make it easier to perceive the sounds, just as, in the domain of color vision, saturated colors are easier to differentiate than muted ones." (Schwartz et al. 1997, p. 259).

More recently Schwartz et al. (2005) have outlined specifically how perceptual asymmetries (in our work and also unexplained asymmetries in their earlier research) support and advance Dispersion- 
Focalization theory and point to a meaningful conceptual link between the evolution and development of vowel systems.

Prior to our NRV framework and related research findings, focal vowels have played an implicit role in developmental theories in that they were often selected to test hypotheses about phonetic development. Clearly there are potential benefits to exploring connections between typological and developmental universals in general and to investigating the explicit role of focalization in guiding vowel development in particular. To advance in this direction we need an applicable metric for quantifying vowel focalization that reveals how this parameter is computed and processed at the behavioral and neural level.

\subsection{Relationship of NRV to other theories/models of speech perception:}

Even before we began our infant vowel research, directional asymmetries were evident in vowel perception studies conducted by Kuhl and colleagues which led them to formulate their Native Language Magnet (NLM) model (Kuhl \& Iverson 1995; Kuhl, Conboy, Coffey-Corina, Padden, Rivera-Gaxiola \& Nelson 2008). In this work infants (and adults) were tested on carefully constructed sets of synthetic vowels that fall within the same vowel category but vary in their category goodness to include prototypic and non-prototypic instances of the same vowel. When infants were tested using variants of a native vowel category they could discriminate a change from a prototypic to non-prototypic vowel but were much less successful when tested in the reverse direction, i.e. a change from a prototypic to a non-prototypic vowel (Grieser \& Kuhl 1989; Kuhl 1991). Kuhl and colleagues interpreted these findings as showing that prototypic vowels in the native language act like perceptual magnets and warp the perceptual space to facilitate access to native categories (Kuhl \& Iverson 1995).

Our first study on English infants' perception of German vowels (Polka \& Werker 1984) was interpretable as a NLM effect. However, as research progressed it soon became clear that the directional asymmetries observed in our work and similar work in other labs could not be explained by NLM. This failure to connect our asymmetries with NLM may be because NLM and NRV have focused on vowel perception patterns at different scales. The NLM model emerged from research exploring how language experience shapes perception of subtle differences in vowel quality that fall within the same vowel category. Our studies with natural speech suggest that directional asymmetries emerge when vowel quality differences are large enough to cross a phonetic boundary. Given these differences in scale, it is possible that both models are correct but apply to different aspects of vowel processing.

From this perspective, directional asymmetries in adults may reveal language-general biases that have shaped the global structure of vowel space (explained by NRV) as well as languagespecific biases that have molded the local internal structure of native vowel categories (described by NLM). To test this view, we developed a set of synthetic vowels to present to French and English adults in both behavioral and neurophysiologic tests (Polka, Molnar, Ménard, Baum \& Steinhauer, submitted). Using the variable linear articulatory model (VLAM, Maeda, 1979) we varied F1 and F2 frequencies to create a series of high vowels that span the back to front dimension from French $\mathrm{l} / \mathrm{/}$ to a more fronted English $/ \mathrm{u} /$ to a front-rounded French $/ \mathrm{y} /$. Note the French $/ \mathrm{u} /$ and $/ \mathrm{y} /$ are phonetically similar to German $/ \mathrm{u} /$ and $/ \mathrm{y} /$. Using identification and rating data from monolingual English and monolingual French adults we selected good and poor instances of French /u/, English /u/ and French /y/ (see Molnar et al., submitted). Preliminary findings reveal asymmetries in ERPs that pattern as predicted by NRV for cross-category vowel pairs and as predicted by NLM for within-category vowel pairs (Polka et al. 2009). Work is in progress to determine if these predictions are also supported in behavioral discrimination of these vowel stimuli. However, 
findings to date suggest that both NRV and NLM are needed to understand how language experience shapes vowel perception.

Recent findings reported by Moon, Lagercrantz \& Kuhl (2009) also suggest that NLM and NRV account for different aspects of infant vowel perception. Using a sucking task, they tested newborn infants' listening preferences for prototypic (P) and non-prototypic (NP) variants of English /i/ and for P and NP prototypic variants of Swedish /y/. They examined P vs NP listening preferences for each vowel in two groups of infants born to English-speaking mothers and two groups of infants born to Swedish-speaking mothers. Consistent with predictions based on NLM, newborns showed a listening preference when they were presented the prototypic vs non-prototypic variants of the non-native but not the native vowel. However, there was also evidence that infants tested on the /i/ variants engaged in more sucking (which activated the presentation of the vowel) compared to infants tested on the /y/ variants (C. Moon, personal correspondence). This difference points to a potential preference for the more peripheral /i/ over /y/, consistent with NRV predictions. It is important to note that this study was not designed to directly assess preference for /i/ vs /y/ within the same infants/; the design focused on testing NLM predictions by evaluating listening preferences for P vs NP variants of the same vowel within the same infants. Assessing both NRV and NLM predictions in newborn listening preferences is an interesting and meaningful step for future research.

Perceptual biases also feature prominently in the PRIMIR framework proposed by Curtin, Byers-Heinlein \& Werker (this volume; see also Werker \& Curtin, 2005). According to PRIMIR there are three dynamic filters that direct processing: initial biases, task demands, and developmental level. The initial biases constrain and guide the infant's attention to cues that specify phonetic contrasts in most languages; focal vowels are included in the list of speech preferences that form the initial bias filter. According to PRIMIR, initial biases are important early in development, and their significance fades as language acquisition advances and the two other dynamic filters, task demands and developmental level, take over. In the expanded version of PRIMIR Curtin et al. (this volume) propose that infants use domain-general statistical learning mechanisms along with a mechanism that operates to compare and contrast information. These two mechanisms are implemented to re-organize initial biases into language-specific phonetic categories.

From our perspective, the NRV framework meshes well with the more comprehensive framework provided by PRIMIR. The NRV framework provides a more elaborated perspective on the early perceptual biases and how they offer an entry into phonetic structure of speech with respect to vowels. Asymmetries in perception can provide insight into processes underlying the reorganization of phonetic perception during acquisition as they are shaped to varying degrees by the dynamic filtering action of the initial biases, developmental level, and task demands. We also fully agree that PRIMIR's proposed "compare-and-contrast mechanism" is essential for speech perception development, but such a mechanism requires a starting unit or salience differential to work with to carry out this function. From our perspective, Natural Referent Vowels are the orienting lights that help access the native vowel system. These stable salient perceptual forms provide a minimal vowel set that the infant can use, via compare and contrast mechanisms described with PRIMIR, to perceptually access additional vowels in their native inventory. Other salience differentials, yet to be discovered, may also be important in launching consonant perception and extracting the native language categories.

\subsection{Taking the next step with the NRV framework}

NRV is a budding conceptual framework. Much additional research is needed to strengthen and elaborate the basic principles of NRV outlined above. Being young there are many challenges 
and opportunities ahead in the development of NRV. One challenge for future research is to formally operationalize formant convergence/focalization as the cue underlying Natural Referent Vowels. A firmer substantive grounding would clearly strengthen our approach, making it possible to formulate and test more specific predictions within the NRV framework. If we are on the right track and infants are using Natural Referent Vowels to build their linguistic systems, then we can use directional asymmetries and vowel perception biases to flesh out effective indices of focalization that support this behavior. As a first step we need further research to confirm and elaborate focalization as the basis for natural referent vowels. Studies using additional vowel pairs that are distinguished by lip rounding (e.g. /I/- /Y/ or / $/ \varepsilon /-/ o e /$ or $/ \Lambda /-/ \propto /$ ) would be informative. If acoustic analyses show less formant convergence for mid or central un-rounded vowels compared to their lip-rounded cognates, then focalization would predict a different asymmetry than a simple F1/F2 location. This outcome would provide an opportunity to test the role of focalization as the defining parameter for natural referent vowels. Studies that involve contrasts among lip-rounded vowels (e.g. /y/- /y/, /œ/ -/ø/) may also provide new insights. Research with synthesized vowels in which formant relationships are systematically manipulated may also help clarify the relationship between formant proximities and perceptual asymmetries.

The NRV framework will also be tested to explicate the origin of natural referent vowels. Research with younger infants can address a number of questions including when (and how) natural referent nowels are formed. Do they reflect an innate bias of the human auditory system or are they acquired through very early experience with speech signals? It will be informative to explore the connection between these early vowel perception biases and infant-directed speech. The acoustics of infant-directed speech, in which the vowel space is expanded relative to adult-directed speech (Kuhl, Andruski, Chistovich, Chistovich, Kozhevnikova, Ryskina, Stolyarova, Sundberg, \& Lacerda 1997; Burnham, Kitamura \& Vollmer-Conna 2002), mirrors the bias for natural reference vowels. This leads to the question of whether infants' perceptual bias favoring natural reference vowels is shaped by the way adults talk to infants, or whether adults play into and exploit this bias to attract the infant's attention, ${ }^{12}$ which at the same time serves to clarify speech for the infant by enhancing extreme vowel contrasts. One way to address this question would be to compare infants' preference for infant-directed speech (Fernald 1985) with and without vowel expansion at different ages.

As we explore the origin of Natural referent vowels, it will also be instructive to assess the potential connection between perceptual biases and emerging production skills. Research with younger infants (below 6 months) will reveal whether a perceptual bias for Natural Referent vowels is in place before infants vocalize or is tied to the emergence of vocal abilities. A recent study on production of corner vowels in the babble of French-learning and English-learning infants suggests a link between the development of infant vowel perception biases and vowel production patterns in older infants (8 to 18 months) (Rvachew, Alhaidary, Mattock, \& Polka, 2008). Specifically we found that the production of $/ \mathrm{u} /$ vowels increases more rapidly in the first year of life for English infants compared to French infants. Similarly, in experiments tracking the listening preference for /u/ vs /y/ (described in section 3.2) we found the /u/ preference to be increasing for English infants and decreasing for French infants within the same age range (see Rvachew et al. 2008 for further discussion). This convergence across studies points to a connection between vowel perception biases and vowel production patterns. Future research that tracks perception and production

\footnotetext{
${ }^{12}$ A study of mothers' infant-direct facial expression (Chong, Werker, Russell \& Carroll 2003) revealed three expression types which express three distinct and consistent emotional messages which can be labelled joy (with spread lips), comfort and caring (with rounded /protruded lips), and excitement/surprise (with a wide open mouth). These three facial gestures correspond to the visemes which go with the extreme vowels /i, $u, a /$.
} 
longitudinally within the same infants is needed to show more directly how perceptual biases are related to the emergence or mastery of new vowel production skills.

Research investigating the species-specificity of vowel perception biases will also provide further insights on the NRV framework (Weiss \& Newport 2006). In 2003, we reviewed studies of vowel perception in infants and in non-human animals. Our comparison revealed a clear difference between human infants (in which asymmetries are a function of the relative position of vowels in the human F1/F2 space) and nonhuman animals (in which asymmetries are almost exclusively a function of the frequency of F2). This suggests that perceptual asymmetries in humans are best described by referring to complex aspects of the acoustic properties of vowels as inhabitants of the human vowel space (see section 4.1), whereas asymmetries in non-human animals are best described by referring to simple aspects of the acoustic properties of vowels as inhabitants of a biologically irrelevant auditory space. Even though the findings for nonhuman animals which Polka \& Bohn (2003) reviewed stem from two different species, avians (blackbirds) and mammals (cats), further research is needed to elucidate the issue of the species-specificity of vowel perception biases.

In conclusion, there are challenges ahead for the NRV framework but also there are also solid reasons for optimism. First, much of the research that led us to formulate this perspective was conducted using vowels in natural syllable productions rather than isolated synthetic vowels. Thus, we do not have to simulate speech in the lab to observe phenomena that are central to our framework. Second, it is encouraging that directional asymmetries have been observed for other types of segments and suprasegmental patterns. Asymmetries have been reported in perception of /r/ vs /l/ (Kuhl, Stevens, Hayashi, Deguchi, Kiritani, \& Iverson, 2005), /b/ vs /v/ (Tsushima, Shiraki, Yoshida \& Sasaki, 2003; Zhang, Imada, Kawakatsu, \& Kuhl, 2006; Altvater-Mackensen \& Fikkert, 2010), stop voicing (Miller \& Eimas, 1996), lexical tone (Harrison, 2000) and for intonation (Hwang, Schafer \& Anderson, 2008). Although it is not clear at present how to explain these findings, they suggest that the general framework of NRV may have a broader application. Finally, the NRV framework generates a range of testable questions that have the potential of establishing exciting links between areas such as infant speech perception and adult non-native speech perception, perception and production in infancy, perceptual biases and vowel systems typology, the acoustic bases of perceptual biases, and the species-specificity of speech perception. We are confident that the NRV framework will motivate research that advances our understanding of speech development and leads to a better understanding of how human phonetic systems have evolved and are grounded in human cognitive and sensory/motor abilities.

\section{Acknowledgements}

This research was supported by research grants from the Natural Sciences \& Engineering Research Council of Canada to the first author, and by grants from the Danish Research Council for the Humanities to the second author.

\section{References}

Altavater-Mackensen, N., Fikkert, P. (2010). The Acquisition of the stop-fricative contrast inp perception and production. Lingua 120, 1898-1909.

Bauer, S.R. \& Benedict, P.K. (1997). Modern Cantonese Phonology, New York: Mouton de Gruyter 
NOTICE: this is the author's version of a work that was accepted for publication in Journal of Phonetics. A definitive version was subsequently published in Journal of Phonetics 39 (2011) 467-478, DOI: 10.1016/j.wocn.2010.08.007

Best, C. T. \& Faber, A. (2000). Developmental increase in infants' discrimination of nonnative vowels that adults assimilate to a single native vowel. Poster presented at the International Conference on Infant Studies, Brighton, UK, 16-19 July 2000.

Bohn, O.-S. \& Bundgaard-Nielsen, R. L. (2008). Second language speech learning with diverse inputs. In: T. Piske \& M. Young-Scholten (Eds.), Input matters in SLA (pp. 207-218). Clevedon, UK: Multilingual Matters.

Bohn, O.-S. \& Polka, L. (2001). Target spectral, dynamic spectral, and duration cues in infant perception of German vowels. Journal of the Acoustical Society of America 110, 504-515.

Bosch, L. \& Sebastián-Gallés, N. (2003). Simultaneous bilingualism and the perception of a language-specific vowel contrast in the first year of life. Language and Speech 46, 217-243

Brown, R. W. \& Lenneberg, E. H. (1954). A study in language and cognition. Journal of Abnormal and Social Psychology 49, 454-462.

Buhr, R. D. (1980). The emergence of vowels in an infant. Journal of Speech and Hearing Research 23, 73-94.

Carney, A.E., Widin, G.P., \& Viemeister, N.F. (1977). Noncategorical perception of stop consonants differing in VOT. Journal of the Acoustical Society of America 62, 961-970.

Chistovich, L. A. \& Lublinskaya, V. V. (1979). The "center of gravity" affect in vowel spectra and critical distance between the formants. Hearing Research 1, 185-195.

Chistovich, L. A. (1985). Central auditory processing of peripheral vowel spectra. Journal of the Acoustical Society of America 77, 789-805.

Chong, S. C. F., Werker, Janet F., Russell, J. A. \& Carroll, J. M. (2003). Three facial expressions mothers direct to their infants. Infant and Child Development 12, 211-232.

Curtin, S. Byers-Heinein, K. \& Werker, J. F. (this volume). Bilingual beginnings as a lens for theory development: PRIMIR in focus.

Fernald, A. (1985). Four-month-old infants prefer to listen to motherese. Infant Behavior and Development 8, 181-195.

Gliga, T., Elsabbagh, M., Andravizou, A. \& Johnson, M. (2009). Faces attract infants' attention in complex displays. Infancy, 14 (5), 550-562.

Grieser, D. \& Kuhl, P. K. (1989). Categorization of speech by infants: Support for speech-sound prototypes. Developmental Psychology 25, 577-588.

Harrison, P. (2000). Acquiring the phonology of lexical tone in infancy. Lingua, 110, 581-66

Hillenbrand, J., Getty, Laura A., Clark, M. J., \& Wheeler, K. (1995). Acoustic characteristics of American English vowels. Journal of the Acoustical Society of America 97, 3099-3111.

Hillenbrand, J., Clark, M. J. \& Houde, R. A. (2000). Some effects of duration on vowel recognition. Journal of the Acoustical Society of America 108, 3013-3022.

Hwang, H., Schafer, A.J. \& Anderson, V.B. ( 2008). Order of presentation asymmetry in intonational contour discrimination in English. Journal of the Acoustical Society of America $124,2497$.

Ishizuka, K., Mugitani, R., Kato, H. \& Amano, S. (2007). Longitudinal developmental changes in spectral peaks of vowels produced by Japanese infants. Journal of the Acoustical Society of America 121, 2272-2282.

Jusczyk, P. W., \& Aslin, R. N. (1995). Infants' detection of the sound patterns of words in fluent speech. Cognitive Psychology, 29, 1-23.

Karypidis, C. (2007). Order effects and vowel decay in short-term memory: The neutralization hypothesis. Proceedings of the $16^{\text {th }}$ International Congress of Phonetic Sciences, 657-660.

Kent, R. D. \& Murray, A. D. (1982). Acoustic features of infant vocalic utterances at 3, 6, and 9 months. Journal of the Acoustical Society of America 72, 353-365.

Kristoffersen, G. (2000). The phonology of Norwegian. Oxford, UK: Oxford Universiy Press. 
Kuhl, P. K. (1991). Human adults and human infants show a "Perceptual Magnet Effect" for the prototypes of speech categories: Monkeys do not. Perception \& Psychophysics 50, 93-107.

Kuhl, P. K., Andruski, J. E., Chistovich, I. A., Chistovich, L. A., Kozhevnikova, E. V., Ryskina, V. L., Stolyarova, E. I., Sundberg, U. \& Lacerda, F. (1997). Cross-language analysis of phonetic units in language addressed to infants. Science 277, 684-686.

Kuhl, P. K., Conboy, B. T., Coffey-Corina, S., Padden, D., Rivera-Gaxiola, M. \& Nelson, T. (2008). Phonetic learning as a pathway to language: New data and native language magnet theory expanded (NLM-e). Philosophical Transactions of the Royal Society B, 363, 9791000.

Kuhl, P. K. \& Iverson, P. (1995). Linguistic experience and the "perceptual magnet effect. In: W. Strange (Ed.), Speech perception and linguistic experience: Theoretical and methodological issues (pp. 121-154). Timonium, MD: York Press.

Kuhl. P.K., Stevens, E. Hayashi, A., Deguchi, T. Kiritani, S. \& Iverson, P. (2005). Infants show a facilitation effect for native language phonetic perception between 6 and 12 months. Developmental Science 9, F13-F21.

Larsson, J. P., Constán, F. V., Sebastián-Gallés, N. \& Deco, G. (2008). Lexical plasticity in early bilinguals does not alter phoneme categories: I. Neurodynamical modeling. Journal of Cognitive Neuroscience 20, 76-94.

Lieberman, P. (1971). Uniquely human: The evolution of speech, thought, and selfless behavior. Cambridge, MA: Harvard University Press.

Lieberman, P. (1980). On the development of vowel production in young children. In: G. YeniKomshian, J. F. Kavanagh. \& C. A. Ferguson (Eds.), Child phonology. Vol. 1: Production (pp. 113-142). New York, NY: Academic Press.

Lindblom, B. \& Engstrand, O. (1989). In what sense is speech quantal? Journal of Phonetics 17, 107-121.

Maeda, S. ( 1979). An articulatory model of the tongue based on a statistical analysis, Journal of the Acoustical Society of America, 65, S22.

Miller, J. L. \& Eimas, P. D. (1996). Internal structure of voicing categories in early infancy. Perception \& Psychophysics 58, 1157-1167.

Molnar, M., \& Polka, L (2004). Vowel preferences in infancy: The role of early language experience. Paper presented at the Boston Child Language Conference, Boston, Mass, November 2004,

Molnar, M., Polka, L., Ménard, L. Baum, S. \& Steinhauer, K. (submitted). Vowel categorization of monolingual and simultaneous bilingual speakers of English and French: Effects of language experience and language mode, Journal of the Acoustical Society of America

Moon, C., Lagercrantz, H. \& Kuhl, P. K. (2009). Newborn infant perception of vowels is affected by ambient language. Journal of the Acoustical Society of America 125, 2778.

Mugitani, R., Fais, L., Kajikawa, S., Werker, J. F. \& Amano, S., (2007). Age-related changes in sensitivity to native phonotactics in Japanese infants. Journal of the Acoustical Society of America 122, 1332-1335.

Nishi, K., Strange, W., Akahane-Yamada, R., Kubi, R. \& Trent-Brown, S. A. (2008). Acoustic and perceptual similarity of Japanese and American English vowels. Journal of the Acoustical Society of America 124, 576-588.

Polka, L. \& Bohn, O.-S. (1996). A cross-language comparison of vowel perception in Englishlearning and German-learning infants. Journal of the Acoustical Society of America 100, 577 592.

Polka, L. \& Bohn, O.-S. (2003). Asymmetries in vowel perception. Speech Communication 41, 221-231. 
Polka, L., Bohn, O.-S. \& Molnar, M. (2005). Natural referent vowels guide the development of vowel perception. Journal of the Acoustical Society of America 117, 2398.

Polka, L. \& Molnar, M. (in preparation). Phonetic biases in early vowel listening preferences. To be submitted to Journal of Speech, Language and Hearing Research.

Polka, L. \& Werker, J. F. (1994). Developmental changes in the perception of non-native vowel contrasts. Journal of Experimental Psychology: Human Perception and Performance 20, 421435.

Polka, L., Sundara, M., Zhaoo, V., Pang, M., \& Ciocca, V. (in preparation). Directional asymmetries in adult vowel perception, To be submitted to Journal of the Acoustical Society of America

Polka, L, Molnar, M., Ménard, L, Baum, S., Steinhauer, K. (submitted). Asymmetries in the MMN response to vowels by French, English, and bilingual adults: evidence for a languageuniversal bias. Proceedings of Meetings in Acoustics

Rosch-Heider, E. H. (1972). Universal in color naming and memory. Journal of Experimental Psychology 93, 10-20.

Rvachew, S., Alhaidarym, A. Mattock, K. \& Polka, L. (2008). Emergence of the corner vowels in the babble produced by infants exposed to Canadian English or Canadian French, Journal of Phonetics, 36, 564-577.

Sebastián-Gallés, N., Echeverria, S. \& Bosch, L. (2005). The influence of initial exposure on lexical representation: Comparing early and simultaneous bilinguals. Journal of Memory and Language 52, 240-255.

Schwartz, J.-L., Abry, C., Boe, L.-J., Ménard, L. \& Valée, N. (2005). Asymmetries in vowel perception, in the context of the Dispersion-Focalisation Theory. Speech Communication 45, 425-434.

Schwartz, J.-L., Boe, L.-J., Vallee, N. \& Abry, C. (1997). The dispersion-focalization theory of vowel systems. Journal of Phonetics 25, 255-286.

Schwartz, J.-L. \& Escudier, P. (1989). A strong evidence for the existence of a large-scae integrated spectral representation in vowel perception. Speech Communication 8, 235-259.

Steinlen, A. K. (2005). The influence of consonantal context on native and nonnatove vowel production: A cross-language study. Tübingen: G. Narr.

Stevens, K. N. (1989). On the quantal nature of speech. Journal of Phonetics 17, 3-46.

Strange, W. (1999). Perception of vowels: Dynamic constancy, In J.M. Pickett (Ed.) The Acoustics of Speech Communication (153-165). Boston, MA: Allyn \& Bacon.

Strange, W., Bohn, O.-S., Trent, S. A. \& Nishi, K. (2004). Acoustic and perceptual similarity of North German and American English vowels. Journal of the Acoustical Society of America $115,1791-1807$

Swoboda, P.J., Morse, P.A., \& Leavitt, L.A. (1976). Continuous vowel discrimination in normal and at risk infants. Child Development, 47, 459-465.

Swoboda, P.J., Kass, J., Morse, P.A., \& Leavitt, L.A. (1978). Memory factors in infant vowel discrimination of normal and at-risk infants, Child Development, 49, 332-339.

Tsushima, T., Shiraki, S. , Yoshida, K., \& Sasaki, M. (2003). On stimulus order effects in discrimination of non-native consonants contrasts. Acoustic Science and Technology, 24, 6.

Vera-Constán, F. \& Sebastián-Gallés, N. (2008a). Asymmetries in speech perception : Perceptual salience at the lexical level. Poster presented at the $29^{\text {th }}$ International Congress of Psychology, Berlin.

Vera-Constán, F. \& Sebastián-Gallés, N. (2008b). Asimetrías en la percepción de vocales : Se mantienen las anclas perceptivas? Paper presented at the 5th SEPEX, San Sebastian, Spain. 
Weiss, D. J. \& Newport, E. L. (2006). Mechanisms underlying language acquisition: Benefits from a comparative approach. Infancy 9, 241-257.

Werker, J. F., \& Curtin, S. (2005). PRIMIR: A developmental framework of infant speech processing. Language Learning and Development, 1(2), 197-234.

Werker, J. F. \& Logan, J. S. (1985). Cross-language evidence for three factors in speech perception. Perception \& Psychophysics 37, 35-44.

Zhang, Y., Imada, T., Kawakatsu, M. \& Kuhl, P.K. (2006). Neural basis of perceptual asymmetry for the stimulus order effect: A cross-language MEG study. Poster presented at Biomag 2006, Vancouver, Canada. 